\title{
Compactly supported biorthogonal two-direction vector-valued wavelet Packet with arbitrary dilation
}

\author{
Lan $\mathrm{LI}^{1, \mathrm{a}}$ \\ ${ }^{1}$ School of Information Engineering, Xi'an University of Arts and Science, Xi'an 710065, P.R China \\ alanli98@126.com
}

Keywords: Birthogonal, Nonseparable,scaling function, Two-direction vector-valued wavelet bases, packets.

Abstract. The notion of two-direction vector-valued wavelet packets which is the generalization of biorthogonal vector-valued wavelet packets is introduced. A method for constructing biorthogonal two-direction vector-valued wavelet packets is obtained and their properties are discussed by means of time- frequency analysis method. Biorthogonality formulas concerning these two-direction vector-valued wavelet packets are established.

\section{Introduction}

Wavelet packets, due to their good properties, have attracted considerable attention. They can be widely applied in science and engineering[1]. Z.Shen [2] generalized the concept of orthogonal wavelet packets to the case of non-orthogonal wavelet packets so that wavelet packets can be used in the case of the spline wavelets. Multivariate nonseparable wavelets have attracted the interest of many mathematicians [3-5]. Although separable wavelet bases have a lot of advantages, they have a number of drawbacks. One way to avoid this is through the construction of nonseparable wavelets. $\operatorname{Li}[6]$ generalized the two-scale refinable equation, established the orthogonality criteria for twodirection refinable function and two-direction wavelet packets. Vector-valued wavelets and multiwavelets are different in the following sense. For example, prefiltering is usually required for discrete multiwavelet transforms but not necessary for discrete valued transforms, especially video images. Therefore, studying two-direction vector-valued wavelets is useful in multiwavelet theory and representions of signals. The objective of this paper is to generalize the concept of biorthogonal vector-valued wavelet packets to biorthogonal two-direction wavelet packets.

\section{Preliminary}

We begin with some notions and notations to be used in this paper. Denote by $\mathrm{R}$ and $\mathrm{C}$ the set of all real and complex numbers, respectively. Denote by $Z$ and $Z_{+}$all integers and nonnegative integers, respectively. Let s be a constant, $s \geq 2, \mathrm{~s} \in Z$. Denote $I_{s}$ and $O$ stand for the $s \times s$ identity matrix and the zero matrix, respectively.

The space $L^{2}\left(R, C^{s}\right)$ is defined to be the set of all vector-valued functions $P(t)$,

$$
L^{2}\left(R, C^{s}\right)=P(\cdot)=\left(\mathrm{p}_{1}(\cdot), \mathrm{p}_{2}(\cdot), \mathrm{L}, p_{r}(\cdot), \mathrm{L}, \mathrm{p}_{s}(\cdot)\right)
$$

where $p_{r}(\cdot)$ denotes the pixel on the $r$ th row at the point $t \cdot\|P\|:=\left(\sum_{l=1}^{s} \int\left|\mathrm{p}_{l}(t)\right|^{2} d t\right)^{1 / 2}$, $\int_{R} P(\mathrm{t}) d x:=\left(\int_{R} p_{l}(\mathrm{t}) d t\right)_{l=1}^{s}$ and Fourier transform is $\quad \hat{H}(\omega):=\int_{R} P(\cdot) \cdot \exp \{-i t \cdot \omega\} d t$. where $t \cdot \omega$ stands for the inner product vectors $t$ and $\omega$. For two vector-valued functions $\mathrm{P}, Q \in L^{2}\left(R, C^{s}\right)$, their symbol inner product is defined to be $\langle P, Q\rangle:=\int_{R} P(x) \mathrm{Q}(x)^{T} d x$, where ${ }^{T}$ means the transpose and the complex conjugate. 


\section{Two-direction vector-valued multiresolution analysis}

The multiresolution analysis method is an important approach to obtain two-direction vector-valued wavelets . Let $\left\{V_{j}\right\}_{j \in Z}$ be a sequence of the closed subspaces $L^{2}\left(R, C^{s}\right)$ satisfying

(i) $\mathrm{L} \subset \mathbf{V}_{-1} \subset \mathbf{V}_{0} \subset \mathbf{V}_{1} \subset \mathrm{L}$;

(ii) $\operatorname{Clos}_{L^{2}\left(R, C^{s}\right)}\left(\bigcup_{j \in Z} V_{j}\right)=L^{2}\left(R, C^{s}\right)$;

(iii) I ${ }_{j \in Z} V_{j}=0$;

(iv) $f(\cdot) \in V_{j} \Leftrightarrow f(\alpha \cdot) \in V_{j+1}, j \in Z$;

(v) There exists a sqeuence $\{\Phi(\cdot-n), \Phi(m-\cdot): n, m \in Z\}$ is a Riesz bases of $V_{0}$.

Then $\left\{V_{j}\right\}_{j \in Z}$ is said to be a two-direction vector-valued multiresolution analysis (TVMRA) with arbitrary dilation. $\Phi(\cdot) \in L^{2}\left(R, C^{r}\right)$ is a corresponding two-direction vector-valued scaling function. Due to the facts that $V_{0} \in V_{1}$, we know that $\Phi(\cdot)$ can be written as

$$
\Phi(\cdot)=\sum_{n \in Z} P_{n}^{+} \Phi(\alpha \cdot-n)+\sum_{n \in Z} P_{n}^{-} \Phi(n-\alpha \cdot)
$$

Where sequence of constant $s \times s$ matrice $\left\{\mathrm{P}_{n}^{+}\right\}_{n \in Z}$ and $\left\{\mathrm{P}_{n}^{-}\right\}_{n \in Z}$ are called the positive-direction vector-valued mask and negative-direction vector-valued mask, respectively.. Applying of the Fourier transform (1), we have

$$
\Phi(\cdot)=P_{0}^{+}\left(\mathrm{e}^{\frac{-i \omega}{\alpha}}\right) \hat{\Phi}\left(\frac{\omega}{\alpha}\right)+P_{0}^{-}\left(e^{\frac{-i w}{\alpha}}\right) \hat{\hat{\Phi}}\left(\frac{\omega}{\alpha}\right)
$$

where $P^{+}(\omega)=\frac{1}{\alpha} P_{n}^{+} \cdot \exp \{-i t \cdot \omega\}, \omega \in R$ and $P^{-}(\omega)=\frac{1}{\alpha} p_{n}^{-} \cdot \exp \{-i t \cdot \omega\}, \omega \in R$ are called positive-direction vector-valued mask symbol and negative-direction vector-valued mask symbol, respectively.

We rewrite (1) as

$$
\Phi(-\cdot)=\sum_{n \in Z} P_{n}^{+} \Phi(-\alpha \cdot-n)+\sum_{n \in Z} P_{n}^{-} \Phi(n+\alpha \cdot)
$$

From (1) and (2), we have

$$
X(\cdot)=\left\{\begin{array}{l}
\Phi(\cdot) \\
\Phi(-\cdot)
\end{array}=\sum_{n}\left|\begin{array}{c}
P_{n}^{+}, P_{n}^{-} \\
P_{-n}^{-}, \mathrm{P}_{-n}^{+}
\end{array}\right| \Phi(\alpha \cdot-n)\right.
$$

Let $W_{n}, n \in Z$ be the orthocomplementary subspace of $V_{n}$. Assume that there exist two two-direction vector-valued functions $\Psi_{v}(t) \in L^{2}\left(R, C^{s}\right), \mathrm{v}=1,2, \mathrm{~L} \alpha-1, \in W_{0} \subset V_{1}$, there exist two finite supported sequences of constant $s \times s$ matrice $\left\{Q_{n}^{+(v)}\right\}_{n \in Z}$ and $\left\{Q_{n}^{-(v)}\right\}_{n \in Z}$ such that

$$
\Psi_{v}(\mathrm{t})=\sum_{n \in Z} Q_{n}^{+(v)} \Phi(\alpha \cdot-n)+\sum_{n \in Z} Q_{n}^{-(v)}(\mathrm{n}-\alpha \cdot), \quad v=1,2, \mathrm{~L} \alpha-1
$$

Implementing the Fourier transform of (4) gives

where

$$
\Psi_{v}(\cdot)=Q_{0}^{+}\left(\mathrm{e}^{\frac{-i \omega}{\alpha}}\right) \hat{\Phi}\left(\frac{\omega}{\alpha}\right)+Q_{0}^{-}\left(e^{\frac{-i w}{\alpha}}\right) \hat{\hat{\Phi}}\left(\frac{\omega}{\alpha}\right), \quad v=1,2, \mathrm{~L} \alpha-1 .
$$

$$
Q^{+(v)}(\omega)=\frac{1}{\alpha} \sum_{n \in Z} Q_{n}^{+} \cdot \exp \{-i t \cdot \omega\}, \quad Q^{-(v)}(\omega)=\frac{1}{\alpha} \sum_{n \in Z} Q_{n}^{-} \cdot \exp \{-i t \cdot \omega\}, \omega \in R, v=1,2, \mathrm{~L} \quad \alpha-1 .
$$

we have

$$
\mathrm{F}_{v}(\cdot)=\left\{\begin{array}{l}
\Psi_{v}(\cdot) \\
\Psi_{v}(-\cdot)
\end{array}=\sum_{n}\left|\begin{array}{l}
Q_{n}^{+(v)}, Q_{n}^{-(v)} \\
Q_{-n}^{-(v)}, \mathrm{Q}_{-n}^{+(v)}
\end{array}\right| \Phi(\alpha \cdot-n)\right.
$$


Similarly, for $\$ \phi$.$) we have$

We rewrite (5) as

$$
\phi(\varphi)=P_{0} \%\left(\mathrm{e}^{\frac{-i \omega}{\alpha}}\right) \hat{\phi} \phi\left(\frac{\omega}{\alpha}\right)+P_{0}\left(e^{\frac{-i w}{\alpha}}\right) \overline{\hat{\phi}}\left(\frac{\omega}{\alpha}\right)
$$

$$
\phi(\rho-\cdot)=\sum_{n \in Z} P_{n}^{+} \phi(\phi-\alpha \cdot-n)+\sum_{n \in Z} P_{n}^{-} \phi\left(\varphi_{n}+\alpha \cdot\right)
$$

From (5) and (6), we have

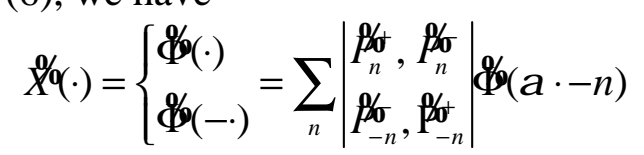

Assume that there exist two two-direction vector-valued functions $\psi_{v}(x) \in L^{2}\left(R, C^{s}\right), \mathrm{v}=1,2, \mathrm{~L} \alpha-1, \in W_{0} \subset V_{1}$, there exist two finite supported sequences of constant $s \times s$ matrice $\left\{\mathscr{Q}_{n}^{(v)}\right\}_{n \in Z}$ and $\left\{\mathscr{Q}_{n}^{(\mathcal{O})}\right\}_{n \in Z}$ such that

$$
\Psi_{v}(\mathrm{t})=\sum_{n \in Z} Q_{n}^{+(v)} \Phi(\alpha \cdot-n)+\sum_{n \in Z} Q_{n}^{-(v)}(\mathrm{n}-\alpha \cdot), \quad v=1,2, \mathrm{~L} \quad \alpha-1
$$

Implementing the Fourier transform of (4) gives

where

$$
\varphi_{v}(\cdot)=B_{0}^{\circ}\left(\mathrm{e}^{\frac{-i \omega}{\alpha}}\right) \hat{\phi}\left(\frac{\omega}{\alpha}\right)+B_{0}^{\circ}\left(e^{\frac{-i w}{\alpha}}\right) \hat{\hat{\phi}}\left(\rho \frac{\omega}{\alpha}\right), \quad \mathrm{v}=1,2, \mathrm{~L} \alpha-1 \text {. }
$$

we have

$$
Q^{+(v)}(\omega)=\frac{1}{\alpha} Q_{n}^{+} \cdot \exp \{-i t \cdot \omega\}, \quad Q^{-(v)}(\omega)=\frac{1}{\alpha} \sum_{n \in Z} Q_{n}^{-} \cdot \exp \{-i t \cdot \omega\}, \omega \in R, v=1,2, \mathrm{~L} \alpha-1
$$

$$
\mathrm{F}_{v} /(\cdot)=\left\{\begin{array}{l}
\Psi_{v}^{\circ}(\cdot) \\
\Psi_{v}(-\cdot)
\end{array}=\sum_{n}\left|\begin{array}{l}
Q_{n}^{+(v)}, Q_{n}^{-(v)} \\
Q_{-n}^{-(v)}, \mathrm{Q}_{-n}^{+(v)}
\end{array}\right| \& \rho \alpha \cdot-n\right)
$$

\section{The properties of biorthogonal two-direction vector-valued wavelet packets}

Set $\quad \Psi_{0}(t)=X(t) \quad, \quad \Psi_{\mu}(t)=F_{\mu}(t), \quad \Psi_{0}(t)=X^{(}(t), \quad \Psi_{\mu}(t)=F_{\mu}(t) \quad, \quad P_{k}^{+(0)}=P_{k}^{+}, P_{k}^{+(\mu)}=Q_{k}^{+(\mu)}$,

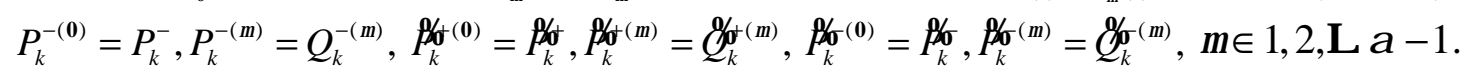

Then, equations (1) and (4) can be written as follows:

$$
\Psi_{\mu}(t)=\sum_{n \in Z} M_{k}^{+(\mu)} \Psi_{0}(\alpha \cdot-k)+\sum_{n \in Z} M_{0}^{-(\mu)} \Psi_{0}(\mathrm{k}-\alpha \cdot), \mu \in 1,2, \mathrm{~L} \alpha-1
$$

Similarly,

$$
\Psi_{\mu}(t)=\sum_{n \in Z} M_{k}^{o(\mu)} \Psi_{0}(\alpha \cdot-k)+\sum_{n \in Z} M_{0}^{\sigma(\mu)} \Psi_{0}(\mathrm{k}-\alpha \cdot), \mu \in 1,2, \mathrm{~L} \alpha-1
$$

For any $\alpha \in Z_{+}$and the given biorthogonal scaling functions $\Psi_{0}(x), \Psi_{0}(x)$, we have

$\Psi_{v}(t)=\Psi_{\alpha \beta+\mu}(t)=\sum_{k \in Z} P_{k}^{+(\mu)} \Psi_{\beta}(\alpha \cdot-k)+\sum_{k \in Z} P_{k}^{-(\mu)} \Psi_{\beta}(\mathrm{k}-\alpha \cdot)$,

$\Psi_{v}(t)=\Psi_{\alpha \beta+\mu}(t)=\sum_{k \in Z} P_{k}^{(\mu)} \Psi_{\beta}(\alpha \cdot-k)+\sum_{k \in Z} P_{k} \gamma^{(\mu)} \Psi_{\beta}^{\circ}(\mathrm{k}-\alpha \cdot)$,

where $\mu \in 0,1,2, \mathrm{~L} \alpha-1, \beta \in Z_{+}$is the unique element such that $v=\alpha \beta+\mu, \mu=0,1,2, \mathrm{~L}, \alpha-1$.

Definition 1 Two-direction vector-valued functions $\left\{\Psi_{\alpha \beta+\mu}(t), \beta \in Z_{+}, \mu=0,1,2, \mathrm{~L}, \alpha-1\right\}$ and $\left\{\Psi_{\alpha \beta+\mu}^{\circ}(t), \beta \in Z_{+}, \mu=0,1,2, \mathrm{~L}, \alpha-1\right\}$ are called biorthogonal two-dierction vector-valued wavelet packets with respect to biorthogonal matrix scaling functions $X(\cdot), \quad X /{ }$.) , respectively, where $\Psi_{\alpha \beta+\mu}(t)$ and $\Psi_{\alpha \beta+\mu}(t)$ are given by (9) and (10), respectively.

Fourier transform for the both sides of equation (9) and (10) 


$$
\begin{aligned}
& \Psi_{\alpha \beta+\mu}^{k}(\omega)=P^{+(\mu)}\left(\frac{\omega}{\alpha}\right) \Psi_{\beta}\left(\frac{\omega}{\alpha}\right)+P^{-(\mu)}\left(\frac{\omega}{\alpha}\right) \hat{\Psi}_{\beta}\left(\frac{\omega}{\alpha}\right), \mu \in 0,1,2, \mathrm{~L}, \alpha-1 \text {. } \\
& \hat{\varphi} \circ_{\alpha \beta+\mu}(\omega)=P^{\prime} /(\mu)\left(\frac{\omega}{\alpha}\right) \hat{\varphi} \%_{\beta}\left(\frac{\omega}{\alpha}\right)+P{ }^{(\mu)}\left(\frac{\omega}{\alpha}\right) \hat{\varphi} \%_{\beta}\left(\frac{\omega}{\alpha}\right), \mu \in 0,1,2, \mathrm{~L}, \alpha-1 .
\end{aligned}
$$

Where

$$
\begin{aligned}
& P^{+(\mu)}(\omega)=\frac{1}{\alpha} \sum_{k \in Z} P_{k}^{+(\mu)} \cdot \exp \{-i k \cdot \omega\}, \quad P^{-(\mu)}(\eta)=\frac{1}{\alpha} \sum_{k \in Z} P_{k}^{-(\mu)} \cdot \exp \{-i k \cdot \eta\}, \mu \in 0,1,2, \mathrm{~L}, \alpha-1 . \\
& \beta \gamma^{(\mu)}(\omega)=\frac{1}{\alpha} \sum_{k \in Z} \beta_{k} \sigma^{(\mu)} \cdot \exp \{-i k \cdot \omega\}, \quad \beta \sigma^{(\mu)}(\eta)=\frac{1}{\alpha} \sum_{k \in Z} \beta_{k}^{\prime(\mu)} \cdot \exp \{-i k \cdot \eta\}, \mu \in 0,1,2, \mathrm{~L}, \alpha-1 .
\end{aligned}
$$

Lemma 1 Let $\Psi(x) \in L^{2}\left(R, C^{s \times s}\right)$, Then it is an biorthogonal vector-valued function if and only if

$$
\sum_{u \in Z} \hat{\Psi}(\omega+2 u \pi) \hat{\varphi} \varphi(\omega+2 u \pi)^{*}=I_{s}, \omega \in R .
$$

Lemma 2. Assume that $\Psi_{v}(x) \in L^{2}\left(R, C^{s}\right)$ and $\Psi_{v}(x) \in L^{2}\left(R, C^{s}\right), v \in 0,1,2, \mathrm{~L}, \alpha-1$ are vector-valued wavelets associated with biorthogonal scaling function $\Psi_{0}(x)$ and $\Psi_{0}(x)$. Then for $\mu, \nu \in \Lambda_{0}$, we have

$$
\sum_{\sigma \in \Lambda_{0}} P^{(\mu)}\left(Q^{-T}(\omega+2 \sigma \pi)\right) P^{(\zeta)}\left(Q^{-T}(\omega+2 \sigma \pi)\right)^{*}=\delta_{\mu, v} I_{s} .
$$

Theorem 1 Assume $\left\{\Psi_{\alpha}(x), \alpha \in Z_{+}^{d}\right\}$ and $\left\{\psi_{\alpha}^{\circ}(x), \alpha \in Z_{+}^{d}\right\}$ are two-direction wavelet packets with respect to biorthogonal two-direction vector-valued function $\Psi_{0}(x)$ and $\Psi_{0}(x)$, respectively.Then, for $\beta \in Z_{+}^{d}, u \in Z^{d}$, we have

$$
\left\langle\Psi_{\alpha \beta+\mu}(\cdot), \varphi_{\alpha \beta+\rho}^{\circ}(\cdot-w)\right\rangle=\delta_{0, w} \delta_{\mu, \rho} I_{s}, \mu, \rho \in 0,1,2, \mathrm{~L}, \alpha-1 .
$$

Proof. Since we set $R$ the following partition: $R=\bigcup_{u \in Z}\left(\frac{1}{\alpha}[0,2 \pi]+2 u \pi\right)$ and we have

$$
\begin{aligned}
& (2 \pi)\left\langle\Psi_{\alpha \beta+\mu}(\cdot), \Psi_{\alpha \beta+\rho}(\cdot-w)\right\rangle=\int_{R} \hat{\Psi}_{\alpha \beta+\mu}(\omega) \Psi_{\alpha \beta+\rho}(\omega)^{*} \cdot e^{i n \cdot \omega} d \omega \\
& =\int_{R^{d}} P^{(\mu)}(1 / \alpha \omega) \hat{\Psi}_{\beta}(1 / \alpha \omega) \cdot \stackrel{\&}{\Psi}{ }_{\beta}(1 / \alpha \omega)^{*} P^{(\rho)}(1 / \alpha \omega)^{*} \cdot \exp \{i n \cdot \omega\} d \omega \\
& =\sum_{u \in Z} \int P^{(\mu)}(1 / \alpha \omega) \hat{\Psi}_{\beta}(1 / \alpha \omega) \cdot \hat{\hat{\varphi}_{\beta}}(1 / \alpha \omega)^{*} P(\otimes)(1 / \alpha \omega)^{*} \cdot e^{i n \cdot \omega} d \omega \\
& =\int_{1 / \alpha^{[0,2 \pi]}} P^{(\mu)}(1 / \alpha \omega)\left[\sum_{w \in Z} \hat{\Psi}_{\beta}(1 / \alpha \omega+2 u \pi) \cdot \dot{\varphi}_{\beta}^{\circ}(1 / \alpha \omega+2 u \pi)^{*}\right] P^{\beta /(0)}(1 / \alpha \omega)^{*} \cdot e^{i w \Upsilon 0} d \omega \\
& =\int_{[0,2 \pi]} \delta_{\mu, \rho} \cdot I_{s} \exp \{i n \cdot \omega\} d \omega=\delta_{0, n} \cdot \delta_{\mu, \rho} \cdot I_{s .}
\end{aligned}
$$

Corollary 1 Suppose $\left\{\Psi_{u}(x), \alpha \in Z_{+}\right\}$and $\left\{\Psi_{u}^{\circ}(x), \alpha \in Z_{+}\right\}$are two-direction vector-valued wavelet packets with respect to biorthogonal two-direction vector-valued function $\Psi_{0}(x)$ and $\Psi_{0}(x)$, respectively. Then, for $v \in Z_{+}^{d}$, we have

$$
\left\langle\Psi_{v}(\cdot), \Psi_{v}^{\circ}(\cdot-w)\right\rangle=\delta_{0, w} I_{s}, w \in Z
$$

Theorem 2. For any $u, v \in Z_{+}$, we have

$$
\left\langle\Psi_{v}(\cdot), \Psi_{u}(\cdot-w)\right\rangle=\delta_{\mathrm{u}, v} \delta_{0, w} I_{s}, w \in Z .
$$

Proof. For $u=v$, we get (15) by Lemma 1. when $u \neq v, \mathrm{u}, v \in \Lambda_{0}$, assuming $u=\alpha u_{1}+\rho_{1}, v=\alpha v_{1}+\mu_{1}$, where $\rho_{1}, \mu_{1} \in 0,1,2, \mathrm{~L}, \alpha-1$. If $v_{1}=u_{1}$, then $\rho_{1} \neq \mu_{1}$. We denote

$$
\begin{aligned}
& (2 \pi)^{d}\left\langle\Psi_{u}(\cdot), \varphi_{v}^{\circ}(\cdot-w)\right\rangle=\hat{\Psi}_{\alpha u_{1}+\rho_{1}}(\omega) \stackrel{\mathrm{Q}}{\Psi}_{\alpha v_{1}+\mu_{1}}^{\mathrm{o}}(\omega)^{*} \cdot \exp \{i u \cdot \omega\} d \omega \\
& =\int_{R^{d}} P^{\left(\rho_{1}\right)}(\xi) \hat{\Psi}_{u_{1}}(\xi) \Psi_{v_{1}}^{\mathcal{Q}}(\xi)^{*} P^{\left.\beta / \phi_{1}\right)}(\xi)^{*} \cdot e^{i w \xi} d \omega
\end{aligned}
$$




$$
\begin{aligned}
& =\int_{[0,2 \pi]} P^{\left(\rho_{1}\right)}(\xi)\left\{\sum_{w \in Z} \hat{\Psi}_{\beta_{1}}(\xi+2 w \pi) \cdot \hat{\varphi} \%_{u_{1}}(\xi+2 w \pi)^{*}\right\} \hat{P}\left(\phi_{1}\right)(\xi)^{*} \cdot e^{i w \cdot \xi} d \xi \\
& =\int_{[0,2 \pi]} \sum_{\sigma \in\{0,1,2, L, \alpha-1 .\}} P^{\left(\rho_{1}\right)}\left[Q^{-T}(\xi+2 \pi w)\right] \cdot P^{\left.\beta / \phi_{1}\right)}\left[Q^{-T}(\xi+2 \pi w)\right]^{*} \cdot \exp \{i w \cdot \omega\} d \omega \\
& =\int_{[0,2 \pi]} \delta_{\rho_{1}, \mu_{1}} I_{s} \cdot \exp \{i w \cdot \omega\} d \omega=O \text {. }
\end{aligned}
$$

If $\alpha_{1} \neq \beta_{1}$, Put $u_{1}=\alpha u_{2}+\rho_{2}, v_{1}=\alpha v_{2}+\mu_{2}$, where $u_{2}, v_{2} \in Z_{+}$, and $v_{2}, \mu_{2} \in 0,1,2, L, \alpha-1$. If $u_{2} \neq v_{2}$, we $\operatorname{order} u_{2}=\alpha u_{3}+\rho_{3}, v_{2}=\alpha v_{3}+\mu_{3}$, where $u_{3}, v_{3} \in Z_{+}, \rho_{3}, \mu_{3} \in\{0,1,2, \mathrm{~L}, \alpha-1\}$. After taking finite steps (denoted by $\kappa$ ), we obtain $u_{\kappa}, v_{\kappa} \in\{0,1,2, \mathrm{~L}, \alpha-1\}$. If $u_{\mathrm{\kappa}}=v_{\mathrm{\kappa}}$, so we have (15) follows. If $u_{\kappa} \neq v_{\mathrm{\kappa}}$, then we have

$$
\left\langle\Psi_{u_{\kappa}}(\cdot), \Psi_{v_{\kappa}}(\cdot-w)\right\rangle=O \Leftrightarrow \sum_{w \in Z} \hat{\Psi}_{u_{\kappa}}(\xi+2 n \pi)^{*} \hat{\Psi} \sigma_{v_{\kappa}}(\xi+2 n \pi)=O, w \in Z
$$

Furthermore, we obtain

$$
\begin{aligned}
& (2 \pi)\left\langle\Psi_{u}(\cdot), \Psi_{v}(\cdot-w)\right\rangle=\int_{R^{d}} \hat{\Psi}_{u}(\omega) \hat{\varphi_{v_{1}}}(\omega)^{*} e^{i w \cdot \omega} d \omega=\int_{R} \hat{\Psi}_{\alpha u_{1}+\rho_{1}}(\eta) \hat{\psi_{\alpha v_{1}+\mu_{1}}}(\eta)^{*} \cdot \exp \{i w \cdot \omega\} d \omega \\
& =\int_{R} P^{\left(\rho_{1}\right)}(\xi) \hat{\Psi}_{u_{1}}(\xi) \hat{\psi} \sigma_{v_{1}}(\xi)^{*} P\left(\phi_{1}\right)(\xi)^{*} \cdot e^{i w \cdot \omega} d \omega \\
& \left.=\int_{R^{d}} P^{\left(\rho_{1}\right)}(\xi) P^{\left(\rho_{2}\right)}\left((1 / \alpha)^{2} \omega\right) \hat{\Psi}_{\alpha_{2}}\left((1 / \alpha)^{2} \omega\right) \cdot \Psi_{v_{2}}(1 / \alpha)^{2} \omega\right)^{*} P^{\left(\mu_{2}\right)}\left((1 / \alpha)^{2} \omega\right)^{*} P^{\left(\mu_{1}\right)}(\xi)^{*} \cdot e^{i w \cdot \omega} d \omega \\
& =\mathrm{L} L \mathrm{~L} L \mathrm{~L} \mathrm{~L} \mathrm{~L} \mathrm{~L}
\end{aligned}
$$

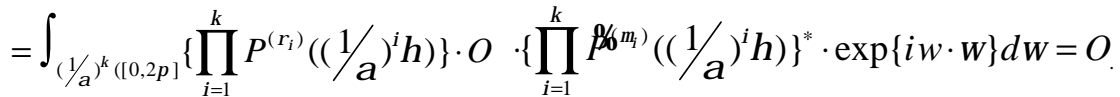

Therefore, for any $\mathrm{u}, v \in Z_{+}$, result (15) follows.

\section{Conclusion}

We present an approach for constructing biorthogonal two-direction vector-valued wavelet and discuss their properties by virtue of time-frequency analysis method, matrix theory and functional analysis method. Two new biorthogonal bases of $L^{2}\left(R, C^{s}\right)$ are obtained.

\section{Acknowledgements}

This work was financially supported by the Project Supported by Natural Science Basic Research Plan in Shaanxi Province of China（Program No.2012JQ1022)

\section{References}

[1] W He and MJ Lai. Examples of bivariate nonseparable compactly supported continuous wavelet,IEEE Trans Signal Process,9(2000)949-953.

[2]Z.Shen, Nontensor product wavelet packets in ${ }^{L_{2}}\left(R^{s}\right)$,SIAM Math. Anal.,26(1995)1061-1074.

[3] W. He and M. J. Lai, Examples of bivariate nonseparable compactly supported orthonormal continous wavelets, IEEE Trans.Image Processing. 9(2000) 949-953.

[4] Y. Z. Li, On the construction of a class of bidimensional nonseparable compactly supported wavelets, Proc. Amer. Math. Soc.133(2005) 3505-3513.

[5] L. Li.ZX Cheng,etl. Construction of a class of trivariate nonseparable compactly supported wavelets with special dilation matrix,Bulletin of the Iranian Mathematical Society, 44(2012)39-54.

[6] L. Li, Z.X Cheng,etl. Biorthogonal two-direction wavelet packets with a positive integer dilation factor, Chinese Journal of Engineering mathematics, 27(2010)901-910. 